\title{
Fermi LAT observations of high energy gamma rays from the Moon
}

\section{Francesco Loparco*}

Università degli Studi di Bari and INFN Sezione di Bari

E-mail: francesco. loparco@ba.infn. it

\section{Mario Nicola Mazziotta}

INFN Sezione di Bari

E-mail: mario.nicola.mazziotta@ba.infn.it

on behalf of the Fermi LAT Collaboration

\author{
Francesco Cerutti \\ CERN \\ E-mail: francesco.ceruttiecern.ch
}

\author{
Alfredo Ferrari \\ CERN \\ E-mail: alfredo.ferrariecern.ch
}

\section{Paola Sala}

INFN Sezione di Milano

E-mail: paola.sala@mi.infn.it

\begin{abstract}
We have measured the gamma-ray emission spectrum of the Moon using the data collected by the Large Area Telescope onboard the Fermi satellite during its first 77 months of operation, in an energy range from $30 \mathrm{MeV}$ up to a few $\mathrm{GeV}$. We have developed a full Monte Carlo simulation describing the interactions of cosmic rays with the Moon surface and the subsequent production of gamma rays using the FLUKA code. The observations can be explained in the framework of this model, where the production of gamma rays is due to the interactions of charged cosmic rays with the surface of the Moon. From the simulation results we have also inferred the cosmic-ray proton spectrum at low energies starting from the gamma-ray measurements. A time evolution study of the gamma-ray emission will be also presented.
\end{abstract}

The 34th International Cosmic Ray Conference,

30 July- 6 August, 2015

The Hague, The Netherlands

\footnotetext{
* Speaker.
} 


\section{Introduction}

High energy gamma rays emitted from the Moon are produced in the inelastic collisions of cosmic-ray nuclei (CRs) with the lunar surface. The gamma-ray emission from the Moon was detected for the first time by the EGRET instrument [1], which operated from 1991 to 2000 onboard the Compton Gamma Ray Observatory (CGRO). A more precise measurement was recently performed using the data from the Large Area Telescope (LAT) onboard the Fermi satellite during its first 2 years of operation [2].

In this work we have evaluated the gamma-ray flux from the Moon using the data collected by the Fermi LAT in its first 6.5 years of operation, from August 2008 to December 2014. We have also performed a study of the time evolution of the gamma-ray flux from the Moon, finding the expected correlation with the solar modulation.

Finally, we have developed a full Monte Carlo simulation of the CR interactions with the Moon surface based on the publicly available FLUKA [5] simulation toolkit, that has been used to infer the solar modulation potential from the Moon gamma-ray data.

\section{Data selection and analysis}

The LAT is a pair conversion gamma-ray telescope, designed to be sensitive in the energy range from $20 \mathrm{MeV}$ to more than $300 \mathrm{GeV}$. A detailed description of the instrument is given in ref. [6]. For this analysis we used a data set corresponding to the first 6.5 years of LAT science operation, from August 2008 to December 2014. The analysis presented has been performed using the newest Pass 8 data [7] and selecting P8_SOURCE photon events starting from a minimum energy of $30 \mathrm{MeV}$.

To study the gamma-ray emission from the Moon we define a signal region, corresponding to the actual position of the Moon, and a background control region. The background is due to the diffuse gamma-ray emission, to the gamma-ray sources that the Moon finds along its path in the sky and to the residual charged CRs erroneously classified as photons.

The signal region is a cone centered on the Moon position, with an energy dependent angular radius given by:

$$
\theta=\sqrt{\left[\theta_{0}\left(E / E_{0}\right)^{-\delta}\right]^{2}+\theta_{\min }^{2}}
$$

where $E$ is the photon energy, $E_{0}=100 \mathrm{MeV}, \theta_{\min }=1^{\circ}, \theta_{0}=5^{\circ}$ and $\delta=0.8$. The energy dependence of the angular radius follows the behavior of the $68 \%$ containment radius of the LAT Point Spread Function (PSF). This choice maximizes the signal to noise ratio. The value of $\theta_{\min }$ accounts for the finite dimension of the Moon, that is seen from the Earth as an extended source of $\sim 0.25^{\circ}$ angular radius. The position of the Moon is obtained from its ephemeris using a software interfaced to the JPL librariesand taking parallax corrections into account.

The background region is a cone of the same angular radius as the signal region, centered on a time-offset position of the Moon. Since the Moon rotates around the Earth with a period of $\sim 28$ days, we chose a time offset value of 14 days (i.e. at a given time the center of the background region is in the position that the Moon will take 14 days later).

For our analysis we selected the time intervals where the LAT was operating in its standard science operation configuration, and was outside the South Atlantic Anomaly (SAA) To avoid 

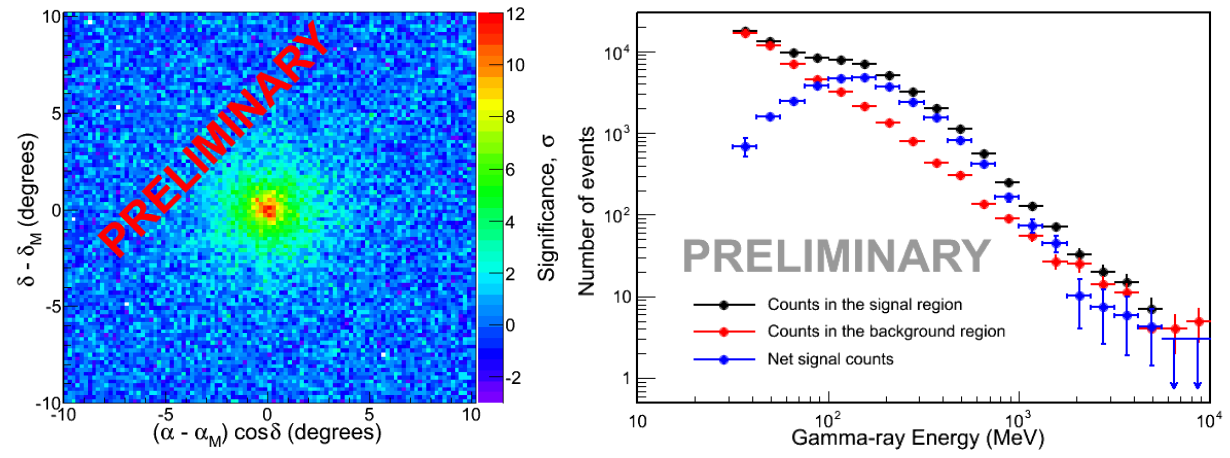

Figure 1: Left: Significance map as a function of right ascension $\alpha$ and declination $\delta$ (the coordinates are centered on the Moon and the pixels correspond to equal solid angles). Right: Photon count distributions in the signal (black circles) and background (red circles) regions. The blue symbols represent the net signal count spectrum, evaluated following the prescriptions of ref. [10].

contamination from the bright limb of the Earth we discarded the data taken during the times when the angular separation between a cone of angular radius $\theta_{\max }$ centered on the Moon direction and the zenith direction exceeded $100^{\circ}$. We also disregarded data taken during the times when the Moon was observed with off-axis angles than $66.4^{\circ}$. To mitigate the systematic uncertainties due to the bright diffuse gamma-ray emission from the Galactic plane, in our analysis we selected only

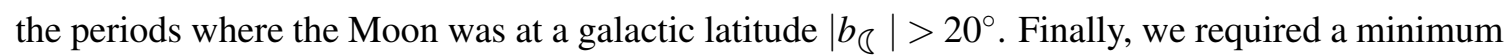
angular distance of $20^{\circ}$ between the Moon and the Sun, and we applied a mask of $20^{\circ}$ angular radius to any bright celestial source in the 2FGL Fermi LAT source catalog [8]. The same analysis cuts were applied to select photons from both the signal and background regions.

In the left panel of fig. 1 we show the significance map of the gamma-ray signal from the Moon, that has been built starting from the counts in the signal and in the background regions, taking into account the livetime ratio between the two regions, and using the formulae in ref. [9], As expected, the significance map is peaked in its center, due to the gamma-ray emission from the Moon.

In the right panel of fig. 1 we show the observed photon count spectra in the signal and background regions, and the net signal count spectrum. To evaluate the net signal counts in each energy bin we followed the bayesian procedure of ref. [10], taking into account the live times of the signal and background regions, and assuming uniform priors for the net signal counts. For each energy bin we evaluated the posterior probability distribution function (PDF) for the signal counts. The central values of the net signal spectrum spectrum correspond to the average values of the PDFs, while the error bars indicate the RMSs. In the energy bins where the signal counts are not significant, only upper limits at $95 \%$ confidence level are shown.

Indicating with $n_{s}\left(E_{i}\right)$ and $n_{b}\left(E_{i}\right)$ the observed counts in the $i$-th energy bin of the signal and of the background regions respectively, we define the likelihood function:

$$
\mathscr{L}\left(\vec{\phi}_{s}, \vec{\phi}_{b} \mid \vec{n}_{s}, \vec{n}_{b}\right)=\prod_{i} e^{-\mu_{s}\left(E_{i}\right)} \frac{\mu_{s}\left(E_{i}\right)^{n_{s}\left(E_{i}\right)}}{n_{s}\left(E_{i}\right) !} \prod_{i} e^{-\mu_{b}\left(E_{i}\right)} \frac{\mu_{b}\left(E_{i}\right)^{n_{b}\left(E_{i}\right)}}{n_{b}\left(E_{i}\right) !}
$$




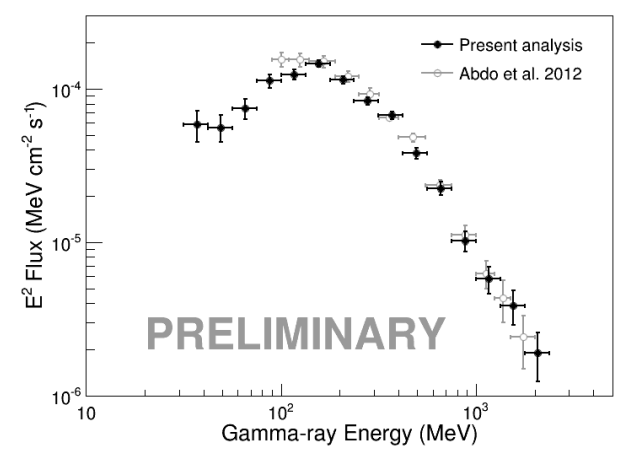

Figure 2: Gamma-ray flux from the Moon as a function of energy. The results of this work (black points) are compared with those published in ref. [2]. Only statistical error bars are shown. The central values of each bin represent the mean flux values, while the error bars represent the RMSs of the corresponding PDFs.

where $\mu_{s}\left(E_{i}\right)$ and $\mu_{b}\left(E_{i}\right)$ are the expected counts in the $i$-th energy bin, that depend on the signal and background gamma-ray fluxes $\phi_{S}(E)$ and $\phi_{b}(E)$. In writing eq. 2.1 we use the vector notation to denote sets of independent quantities defined in the various energy bins (i.e. $\vec{\phi}_{s}=$ $\left(\phi_{s}\left(E_{1}\right), \phi_{s}\left(E_{2}\right), \ldots,\right)$ etc.). The expected counts depend on the signal and background gamma-ray fluxes through the following relations:

$$
\begin{gathered}
\mu_{s}\left(E_{i}\right)=\sum_{j} P_{s}\left(E_{i} \mid E_{j}\right)\left[\phi_{s}\left(E_{j}\right)+\phi_{b}\left(E_{j}\right)\right] A t_{s} \Delta E_{j} \\
\mu_{b}\left(E_{i}\right)=\sum_{j} P_{b}\left(E_{i} \mid E_{j}\right) \phi_{b}\left(E_{j}\right) A t_{b} \Delta E_{j} .
\end{gathered}
$$

In eqs. 2.2 and $2.3 P_{s}\left(E_{i} \mid E_{j}\right)$ and $P_{b}\left(E_{i} \mid E_{j}\right)$ are the smearing matrices in the signal and background regions respectively, i.e. the probabilities that a photon of energy $E_{j}$ is observed with energy $E_{i}$, and are evaluated from a full Monte Carlo simulation of the instrument, that accounts for the pointing history of the two regions; $A$ is the acceptance of the surface used for the generation of the events in the Monte Carlo simulation; $t_{s}$ and $t_{b}$ are the live times of the signal and background regions respectively.

To evaluate the signal and background gamma-ray fluxes we used the software toolkit BAT [11], that implements a bayesian algorithm based on the Markov Chain Monte Carlo (MCMC) to find the parameters that maximize the likelihood of a given model. In our case we used BAT to evaluate, starting from the observed count distributions in the signal and background regions, the posterior PDFs for both the signal and background gamma-ray fluxes $\phi_{s}\left(E_{j}\right)$ and $\phi_{b}\left(E_{j}\right)$.

The reconstructed gamma-ray fluxes from the Moon are shown in fig. 2 and are compared with the fluxes published in ref. [2]. The points shown in the plot correspond to the mean values of the PDFs on the signal fluxes in each bin, while the error bars indicate the RMSs. The function $E^{2} \phi_{\gamma}(E)$ is peaked at about $150 \mathrm{MeV}$ and drops rapidly with increasing energy. The results of this measurement are consistent with those of ref. [2] in the energy range above $150 \mathrm{MeV}$. The discrepancies in the region below $150 \mathrm{MeV}$ are due to the solar modulation effect on CRs. In fact, the data in ref. [2] were taken from August 2008 to August 2010, at the beginning of Solar Cycle 

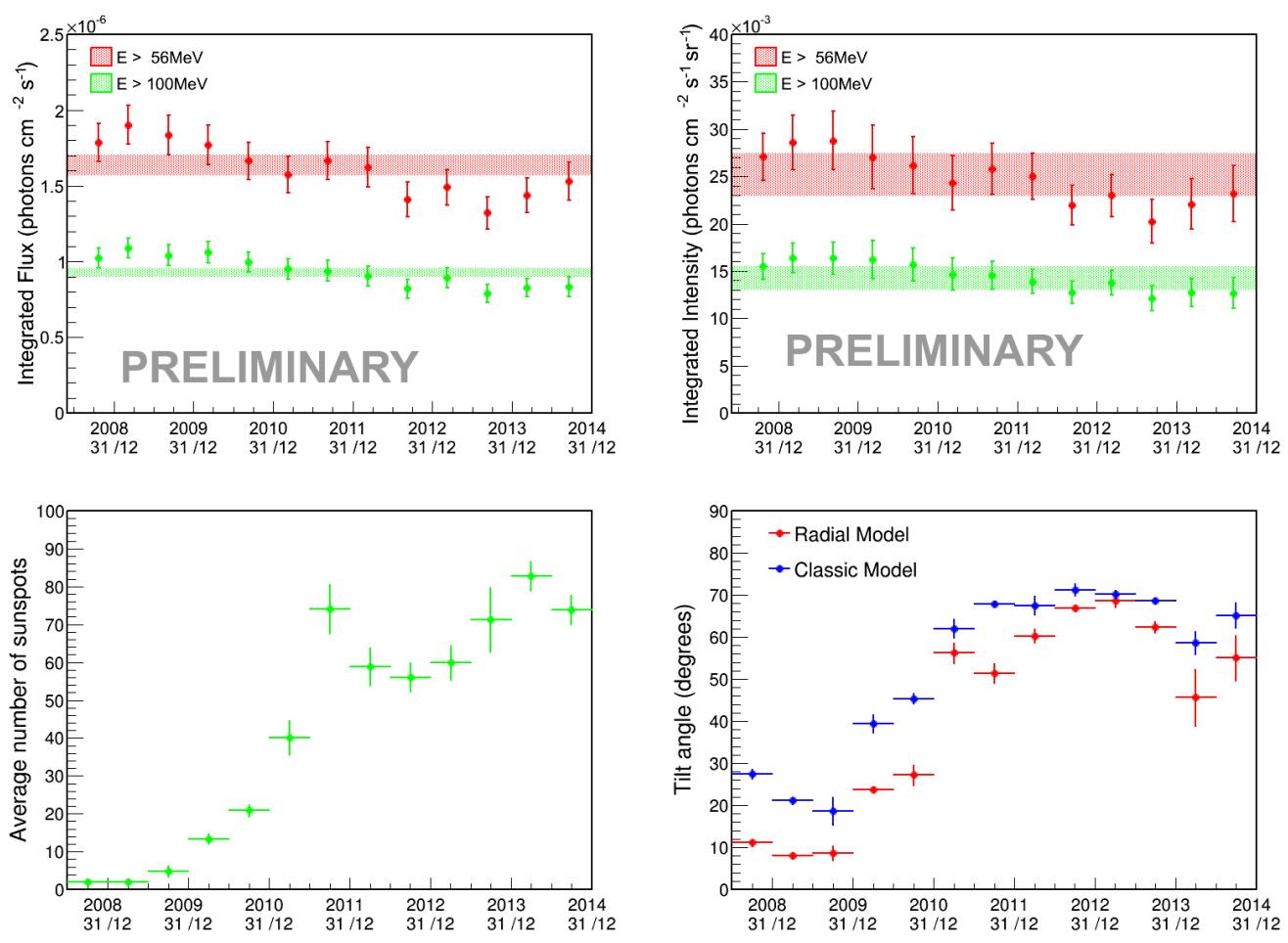

Figure 3: Top left: Time evolution of the gamma-ray flux from the Moon above 56 (red) and $100 \mathrm{MeV}$ (green). The shaded areas indicate the average values over the whole data taking period. Top right: Time evolution of the gamma-ray intensity from the Moon. Bottom left: Time evolution of the average number of sunspots. The data are taken from [12] and refer to the Brussels International Sunspot Number. Bottom right: Time evolution of the average heliospheric current sheet tilt angle for two potential field models. The blue points correspond to the model in ref.[14] ("classic model"), while the red points correspond to the model in ref. [15] ("radial model").

24, when the solar activity was at its minimum. On the other hand, the data presented here cover the time lapse from August 2008 to December 2014, when an increase of the solar activity was detected.

\subsection{Time evolution studies}

To study the time evolution of the gamma-ray emission from the Moon, we divided the data set into smaller samples corresponding to periods of 6 months duration, with the exception of the first one, corresponding to the 5 months from August 2008 to December 2008, and we analyzed separately each of these samples. The top left plot in fig. 3 shows the time evolution of the gamma-ray flux from the Moon above 56 and $100 \mathrm{MeV}$. The fluxes in the various time intervals are compared with the average value, calculated over the whole data taking period.

The flux detected by the LAT is related to the intensity of gamma rays emitted from the Moon by the following equation:

$$
\phi_{\gamma}\left(E_{\gamma}\right)=\frac{\pi R_{\overparen{Q}}^{2}}{d^{2}} I_{\gamma}\left(E_{\gamma}\right)
$$



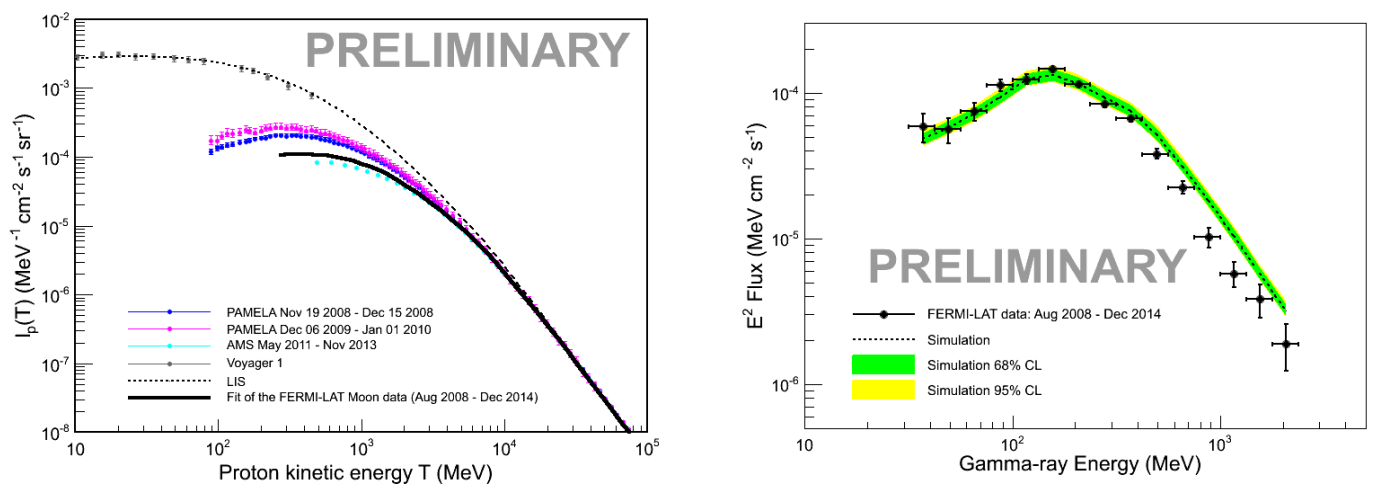

Figure 4: Left panel: CR proton spectrum obtained from the best fit of the Fermi LAT Moon gammaray data. The result of the fit (continuous black line) is compared with the proton measurements taken by PAMELA [3] in 2008 (blue points) and 2009 (purple points) and with the AMS-02 [4] data (cyan points). The plot shows also the LIS (dashed black line) and the Voyager 1 data [21] (grey points). Right panel: Gamma-ray flux from the Moon as a function of energy. The results shown in fig. 2 are compared with those of the fit. The dotted line represents the average gamma-ray spectrum obtained from the fit, assuming that the Moon-LAT distance is equal to its average value during the whole data taking period. The green and yellow bands represent respectively the $68 \%$ and $95 \%$ confidence level central intervals, and are obtained taking into account the variations of the Moon-LAT distance during the data taking period.

where $R_{\overparen{C}}=1737.1 \mathrm{~km}$ is the radius of the Moon and $d$ is the LAT-Moon distance. The top right plot in fig. 3 shows the time evolution of the gamma-ray intensity, evaluated starting from the flux and assuming for $d$ the average value of the LAT-Moon distance. During the data taking period the LAT-Moon distance varied in the range from about $3.4 \times 10^{5} \mathrm{~km}$ to $4.1 \times 10^{5} \mathrm{~km}$, because of the orbital motions of the Moon (with a period $\approx 28$ days) and of the LAT (with a period $\approx 1.5$ hours). These distance variations were taken into account in evaluating the errors on the intensities shown in the plot.

In the bottom left plot in fig. 3 it is shown the time evolution of the average number of sunspots during the period considered for this analysis. The plot was built using the data distributed by the Space Weather Prediction Center [12]. Finally, the bottom right plot in fig. 3 shows the time evolution of the average tilt angle of the heliospheric current sheet.

A comparison of all the plots in fig. 3 shows that the gamma-ray emission from the Moon seems to be anticorrelated to the number of sunspots and to the heliospheric current sheet tilt angle. This result is expected, since gamma rays are produced in the interactions of primary CRs with the surface of the Moon, and therefore their flux must be affected by solar modulation.

\section{Study of the solar modulation potential}

We have developed a Monte Carlo simulation of the interactions of CRs with the surface of the Moon to evaluate, starting from the lunar gamma-ray data, the local intensity spectrum of CR protons. We used the FLUKA [5] simulation code to evaluate the yields of gamma rays produced by $\mathrm{CR}$ protons interacting with the Moon surface. 
In our model we describe the Moon as a perfect sphere of radius $R_{\mathbb{8}}=1737.1 \mathrm{~km}$, consisting of a mixture of different oxides $\left(48.0 \% \mathrm{SiO}_{2}, 4.1 \% \mathrm{FeO}, 11.6 \% \mathrm{CaO}, 24.9 \% \mathrm{Al}_{2} \mathrm{O}_{3}, 9.9 \% \mathrm{MgO}\right.$, $0.7 \% \mathrm{TiO}_{2}$ and $0.6 \% \mathrm{Na}_{2} \mathrm{O}$ ) with a density $\rho=3.01 \mathrm{~g} / \mathrm{cm}^{3}$. The choice of this composition is the result of ana analysis of the lunar gamma-ray emission data in the periods when direct measurements of the primary CR proton spectrum were taken by the PAMELA [3] (2008-09) and AMS [4] (May 2011-November 2013) experiments. We tested several composition models available in literature $([16,18,17])$ and we noticed that, to reproduce the measured lunar gamma-ray fluxes starting from the primary CR proton intensity spectra, a lighter composition of the lunar surface with respect to those models should be assumed. Hence we developed a custom composition model that differs from the models in refs. $[16,18,17]$ because the fractions of light oxides - mainly $\mathrm{SiO}_{2}$ - are slightly higher (and, conversely, the fractions of heavy oxides - mainly $\mathrm{FeO}$ - are slightly lower) [19].

The gamma-ray intensity spectrum from the Moon is related to the intensity spectrum of CR protons in the solar system by the following relation:

$$
I_{\gamma}\left(E_{\gamma}\right)=\int Y\left(E_{\gamma} \mid T_{p}\right) I_{p}\left(T_{p}\right) d T_{p}
$$

where $Y\left(E_{\gamma} \mid T_{p}\right)$ is yield of gamma rays of energy $E_{\gamma}$ produced by protons of kinetic energy $T_{p}$ and $I_{p}\left(T_{p}\right)$ is the CR proton intensity spectrum in the Solar System. The latter can be evaluated from the Local Interstellar Spectrum (LIS) in the framework of the force field approximation as:

$$
I\left(T_{p}\right)=I_{L I S}\left(T_{p}+\Phi\right) \times \frac{T_{p}\left(T_{p}+2 m_{p}\right)}{\left(T_{p}+\Phi\right)\left(T_{p}+\Phi+2 m_{p}\right)}
$$

where $m_{p}$ is the proton mass and $\Phi$ is the solar modulation potential.

In this analysis we assumed a model of the LIS [20], and we fitted our data leaving the solar modulation potential as a free parameter. The fit procedure is based on BAT, and is similar to the one described in section 2. In this case the gamma-ray signal fluxes in the various energy bins are correlated, and are calculated from the cosmic-ray proton intensity $I\left(T_{p}\right)$, while the parameters to be fitted are the background photon fluxes $\vec{\phi}_{b}$ and the solar modulation potential $\Phi$.

The fit procedure results into an average value of the solar modulation potential of (502 \pm 11) $\mathrm{MeV}$ during the whole data acquisition period. The left panel of fig. 4 shows the fitted CR proton intensity spectrum, compared with the results of the direct measurements performed by PAMELA and by AMS. The CR proton spectrum inferred from this analysis is consistent with the results from direct measurements and lies in the region between the PAMELA and the AMS data. The gamma-ray spectrum obtained from the fit is shown in the right panel of fig. 4, where it is compared with the results from the data analysis discussed in section 2 . The central confidence belts at the levels of $68 \%$ and $95 \%$ have been evaluated taking into account the variations of the Moon-LAT distance during the data taking period.

\section{Conclusions}

We measured the fluxes of gamma rays produced by the interactions of charged CRs impinging on the Moon surface using a sample of data collected by the Fermi LAT from August 2008 to December 2014. 
We also developed a full Monte Carlo simulation of the interactions of CR protons with the Moon using the FLUKA simulation code to evaluate the gamma-ray yields. Using the simulation we have inferred the local CR proton intensity spectrum from the Moon gamma-ray spectrum in the framework of the force field approximation.

\section{Acknowledgements}

The Fermi-LAT Collaboration acknowledges support for LAT development, operation and data analysis from NASA and DOE (United States), CEA/Irfu and IN2P3/CNRS (France), ASI and INFN (Italy), MEXT, KEK, and JAXA (Japan), and the K.A. Wallenberg Foundation, the Swedish Research Council and the National Space Board (Sweden). Science analysis support in the operations phase from INAF (Italy) and CNES (France) is also gratefully acknowledged.

\section{References}

[1] D. J. Thompson et al., J. Geophys. Res. 102 (1997), 14735.

[2] A. A. Abdo et al., Astrophys. J. 758 (2012), 140.

[3] O. Adriani et al., Astrophys. J. 765, 91 (2013) [arXiv:1301.4108 [astro-ph.HE]].

[4] M. Aguilar el al., Phys. Rev. Lett. 114, 171103 (2015)

[5] G. Battistoni et al., Proc. of the Hadronic Shower Simulation Workshop 2006, AIP Conf. Proc. 896 (2007), 31; A. Ferrari et al., CERN-2005-10 (2005), INFN/TC_05/11, SLAC-R-773; http://www. fluka.org

[6] W. B. Atwood et al., Astrophys. J. 697 (2009), 1071.

[7] W. B. Atwood et al. (Fermi-LAT Collaboration), 2012 Fermi Symposium proceedings, eConf C121028 (2013), [arXiv:1303.3514 [astro-ph.IM]]

[8] P. Nolan et al., Astrophys. J. Supp. 199 (2012), 31.

[9] T. Li and Y. Ma, Astrophys. J. 272 (1983), 317.

[10] F. Loparco and M. N. Mazziotta, Nucl. Inst. Meth. A646 (2011), 167.

[11] A. Caldwell, D. Kollar, K. Krøninger, Comput. Phys. Commun. 180 (2009), 2197.

[12] http://swpc.nooa.gov/products/solar-cycle-progression

[13] http://wso.stanford.edu/Tilts.html

[14] J. T. Hoeksema in Solar Wind Seven Colloquium, ed. Marsch \& R. Schwenn, Pergamon Press New York (1991).

[15] X. Zhao and J. T. Hoeksema, Adv. Space Res. 16 (1995), 181.

[16] I. V. Moskalenko and T. A. Porter, Astrophys. J. 670 (2007), 1467.

[17] A. L. Turkevich, The Moon 8 (1973), 365.

[18] S. Ota et eal., Earth Planets Space 63 (2011), 25-35.

[19] The Fermi LAT Collaboration, in preparation.

[20] M. N. Mazziotta et al., in preparation.

[21] E.C. Stone et al., Science 341 (2013), 150 